\title{
Dynamics of localized waves: Pulsed microwave transmissions in quasi-one-dimensional media
}

\author{
Z. Q. Zhang, ${ }^{1}$ A. A. Chabanov, ${ }^{2}$ S. K. Cheung, ${ }^{1}$ C. H. Wong, ${ }^{1}$ and A. Z. Genack ${ }^{3}$ \\ ${ }^{1}$ Department of Physics, Hong Kong University of Science and Technology, Clear Water Bay, Kowloon, Hong Kong \\ ${ }^{2}$ Department of Physics and Astronomy, University of Texas at San Antonio, San Antonio, Texas 78249, USA \\ ${ }^{3}$ Department of Physics, Queens College, City University of New York, Flushing, New York 11367, USA
}

(Received 3 March 2008; published 9 April 2009)

\begin{abstract}
We have measured pulsed microwave transmission through quasi-one-dimensional (quasi-1D) samples with lengths up to three times the localization length as determined from measurements of the variance of intensity fluctuations. Measurements are analyzed using four complementary approaches, each appropriate in a specific time range: (i) diffusion theory; (ii) self-consistent localization theory (SCLT) with a renormalized diffusion coefficient in space and frequency, $D(z, \Omega)$; (iii) a dynamic single parameter scaling (DSPS) model, which reflects the decay of localized modes which do not overlap in space and frequency; and (iv) simulations of 1D random media. For times up to twice the diffusion time $\tau_{D}$, diffusion theory gives an excellent fit to the data. For times up to $4 \tau_{D}$, the slowing of the decay rate of transmission is in accord with SCLT. For longer times, transmission decays more slowly than the predictions of the SCLT, indicating the inability of this modified diffusion theory to capture the decay of long-lived localized states. Beyond the Heisenberg time, the decay rate approaches the predictions of the DSPS model, reflecting the increasing proportion of wave energy in longlived localized states. The decay rates obtained from 1D simulations are then in good agreement with measurements in quasi-1D samples and coincide with decay rates given by the DSPS model.
\end{abstract}

DOI: 10.1103/PhysRevB.79.144203

PACS number(s): 42.25.Dd, 05.60.-k, 42.25.Bs, 73.23.-b

\section{INTRODUCTION}

The theory of localization was developed in the context of electronic conduction. ${ }^{1,2}$ Because of electron-electron interactions, however, the localization transition for electrons is not a pure single-particle Anderson transition. Localization is also possible for classical waves in acoustics and optics (see, for example, Refs. 3 and 4). However, the exponential decay of average transmission expected for localized waves is also found for diffusive waves in the presence of absorption. The description of localization is made all the more complex by inelastic scattering, since the impact of inelastic scattering grows with path length and waves following trajectories of all lengths contribute to the transmission. Since even low absorption levels can affect transmission when the dwell time of the wave in the sample is long, as it is in strongly scattering samples, it is difficult to determine the localization length from measurements of the exponential scaling of transmission. 5,6 Similarly, the rounding of the coherentbackscattering (CBS) peak cannot unequivocally establish localization since it is produced by both absorption and localization. ${ }^{6-11}$ However, localization can be disentangled from absorption using pulsed measurements since all paths within the sample at a given delay time are of the same length. The relative weights of paths are therefore unaffected by absorption. ${ }^{12-15}$ Since pulsed measurements have not been feasible for electrons, theoretical studies on electron localization have focused on static transport.

Though dynamical studies bring a new dimension to studies of wave localization, it is essential that dynamics be understood in the context of static transport. Only then can the degree of localization in the sample studied be established. The increasing suppression of transport with time delay from an exciting pulse, due to the increasing impact of weak localization with path length, becomes more significant as the localization transition is approached but can also occur in diffusive samples. ${ }^{14}$ The analysis of wave dynamics should therefore start with the determination of the degree of steadystate localization within sample. In the absence of inelastic processes, the degree of localization can be expressed by the dimensionless conductance ${ }^{2} g$, or equivalently by the Thouless number, ${ }^{16} \delta=\delta \nu / \Delta \nu$. $1 / \Delta \nu$ gives the density of states of the sample as a whole, and $\delta \nu$ is the field correlation frequency. ${ }^{17}$ Therefore, the Thouless number is also a measure of the degree of spectral overlap. The localization threshold corresponds to the condition that $g=\delta=1$. $^{16}$ The localization threshold can also be expressed in terms of dynamic parameters as the condition that the Thouless time equals the Heisenberg time, $\tau_{\mathrm{Th}}=\tau_{H}$. Here, $\tau_{\mathrm{Th}}=1 / \delta \nu$ $=\pi^{2} \tau_{D}$, and $\tau_{H}=1 / \Delta \nu$ is the time required to visit each coherence volume of the sample. In the presence of absorption, transmission drops, while the linewidth increases, so that $g$ and $\delta$ are affected by absorption in different ways and their values are no longer reliable measures of localization. Another measure of localization is the variance of transmitted intensity between incident and outgoing modes, $a$ and $b$, respectively, normalized by the ensemble average value, $s_{a b}$ $=T_{a b} /\left\langle T_{a b}\right\rangle$. For simplicity, we shall often denote $T_{a b}$ as $I$. In the diffusive limit, the field is a Gaussian random variable and $\operatorname{var}\left(s_{a b}\right)=1$, whereas at the localization threshold, $\operatorname{var}\left(s_{a b}\right) \simeq 7 / 3(2.33) .{ }^{13,18}$ In quasi-one-dimensional (quasi1D) samples, $\operatorname{var}\left(s_{a b}\right)=2 \operatorname{var}\left(s_{a}\right)+1,{ }^{18,19}$ where $s_{a}$ is the total transmission normalized by its average over a random ensemble of statistically equivalent samples, $s_{a}=T_{a} /\left\langle T_{a}\right\rangle$, for an incident mode $a$. The localization threshold is at $\operatorname{var}\left(s_{a}\right)$ $\simeq 2 / 3 g=2 / 3$ when absorption is absent, but is not significantly affected by absorption.

Far from the localization threshold, measurements of the average pulsed transmission $\langle I(t)\rangle$ in opaque samples have generally been well described by diffusion theory. ${ }^{20}$ For times greater than the diffusion time $\tau_{D}$, higher diffusion 
modes decay rapidly, leaving energy in the lowest diffusion mode with a decay rate approaching the constant value, $1 / \tau_{D}=\pi^{2} D /\left(L+2 z_{0}\right)^{2} \cdot{ }^{20}$ Here, $D$ is the diffusion coefficient, $L$ is the sample length, and $z_{0}$ is the distance beyond the boundary at which the intensity within the sample extrapolates to zero. ${ }^{21}$ However, a progressive suppression of the decay rate of transmission has been observed in recent microwave experiments ${ }^{14}$ in samples which were determined to be firmly on the diffusive side of the localization transition from measurements of $\operatorname{var}\left(s_{a b}\right)$. The response to a pulse was found from spectral measurements of transmitted field in a sample of randomly positioned alumina spheres at low concentrations, within a quasi-1D geometry, in a frequency range between two Mie resonances of the spheres. ${ }^{14}$ Measurements were made in samples with lengths for which $\operatorname{var}\left(s_{a b}\right)$ was between 1.18 and 1.5.

The slowing decay of $\langle I(t)\rangle$ for diffusive waves reflects the increasing prominence of longer-lived modes, ${ }^{14}$ which are more remote from the sample boundaries and more sharply peaked within the sample. ${ }^{3,22-25}$ This is associated with the increasing enhancement of weak localization with longer path length due to the scattering of the wave as it crosses over its trajectory. ${ }^{26}$ The impact of localization on electron dynamics at $T=0$ had been calculated using diagrammatic, nonlinear $\sigma$, and supersymmetry approaches. ${ }^{22,27}$ Vollhardt and Wölfle ${ }^{28}$ (VW) developed a self-consistent diagrammatic theory of localization within a medium in terms of a frequency-dependent renormalized diffusion coefficient $D(\Omega)$. To be consistent, Van Tiggelen et al. ${ }^{29}$ argued that $D$ must also be a function of depth within a bounded sample, $D(z, \Omega)$. Skipetrov and Van Tiggelen ${ }^{30,31}$ used self-consistent localization theory (SCLT) to describe waves near the mobility edge for $t<\tau_{H}$ in quasi-1D (Ref. 30) and slab geometries. ${ }^{31}$ They described ${ }^{30}$ key features observed in microwave measurements ${ }^{14}$ for diffusive waves in quasi-onedimension and found a $1 / t^{2}$ falloff in reflection for localized waves.

Suppressed pulsed transmission was also observed by Störzer et $a l .{ }^{15}$ in optical measurements in a titania powder slab. Measurements of CBS gave a value of $k \ell$, where $k$ is the wave number and $\ell$ is the transport mean free path, as small as 2.5 . The value of $k \ell$ inside the sample is presumably smaller but still appears to be larger than unity, which is the Ioffe-Regel criterion for localization in three dimensions. ${ }^{32}$ The analysis of the measured $\langle I(t)\rangle$ was based on a phenomenological theory incorporating a temporally varying diffusion coefficient $D(t) .{ }^{33}$ Such a phenomenological theory does not consider the retardation of localization effects in the renormalization of the diffusion coefficient. The influence of retardation on an effective diffusion coefficient can be captured, however, by a renormalization of the diffusion coefficient in frequency, i.e., $D(\Omega) .^{26,28-30}$ Statistical measurements of the nature of propagation were not reported, so that the precise degree of correlation and associated localization was not ascertained.

Measurements of the dynamics of localized waves in which static localization was established were first reported for microwave radiation in quasi-1D samples in Ref. 34. Those results are recapitulated and elaborated here. Recently, $\mathrm{Hu}$ et al. ${ }^{35}$ reported suppressed leakage rate in measurement of pulsed ultrasound transmission through a slab of aluminum beads. A single sample was studied because the delicate balancing to properly sinter the sample was only achieved in a single sample. Intensity statistics could be determined, however, by measuring intensity across the sample surface over a range of frequencies. These measurements gave $\operatorname{var}\left(s_{a b}\right)=2.6>2.33$ in one frequency range, indicating that ultrasound propagation was close to the Anderson localization transition on the localized side. Pulsed decay in the sample was well fitted by SCLT.

In this paper, we present microwave measurements and analysis of dynamic transmission for localized waves in quasi-1D samples with lengths greater than the average localization length, $L>\bar{\xi}$. The localization length was determined from measurements of variance of intensity fluctuations, as discussed in Appendix A. Measurements are analyzed in different time ranges using four different approaches. For times up to twice the peak arrival time, $t_{p}$ $\sim \tau_{D},\langle I(t)\rangle$ is well described by classical diffusion theory. For $t>2 \tau_{D}$, however, measurements fall more slowly than predicted by diffusion theory. For $2 \tau_{D}<t<4 \tau_{D}$, measurements are in accord with SCLT, which includes a positionand frequency-dependent diffusion coefficient $D(z, \Omega){ }^{30,31}$ For $t>4 \tau_{D}$, the transmission decay rate $\Lambda(t)=$ $-[d\langle I(t)\rangle / d t] /\langle I(t)\rangle=-d \ln \langle I(t)\rangle / d t \quad$ is increasingly suppressed by localization up to a factor of nearly 3 relative to the early diffusive decay rate $1 / \tau_{D}$ in the samples measured. This leads to an enhancement in the transmitted flux by a factor of $10^{5}$ at a time of $20 t_{p}$. However, the SCLT predicts a faster decay rate than observed in the experiment. The breakdown of SCLT reflects the inability of a modified diffusion theory to capture the decay of long-lived states in the medium. In order to model the long-time decay of transmission, we develop a dynamic single parameter scaling (DSPS) model, which is based on the key element of steady-state single parameter scaling (SPS), a Gaussian distribution of Lyapunov exponents, $\gamma=1 / 2 \xi$, with average $\bar{\gamma}=1 / 2 \bar{\xi}$ and $\operatorname{var}(\gamma)=\bar{\gamma} / L{ }^{36}$ As is the case of the SPS model, the DSPS model assumes that all modes are exponentially localized and ignores the spectral and spatial overlap of modes that leads to the formation of more rapidly decaying necklace states. For this reason, we do not expect this model to accurately represent transmission at early times, but explore the degree to which the model is able to catch aspects of decay at times long enough that dynamics is dominated by long-lived modes. Beyond the Heisenberg time, the decay rate approaches the predictions of the DSPS model. The decay rates obtained from 1D simulations are also found to be in good agreement with measurements in quasi-1D samples and to coincide with decay rates given by the DSPS model. This indicates that dynamics at late times is determined by the similar distributions of $\xi$ in $1 \mathrm{D}$ and quasi-1D samples. In contrast, the short-time dynamics is affected by mode overlap which may differ sharply in 1D and quasi-1D samples. 1D simulations give a peak in the decay rate which is higher and peaks later than in measurements in quasi-1D samples. The diffusionlike delay of the transmission peak reflects the impact of short-lived, spectrally overlapping, quasiextended modes, described by Pendry ${ }^{23}$ as necklace states. ${ }^{23-25}$ These 

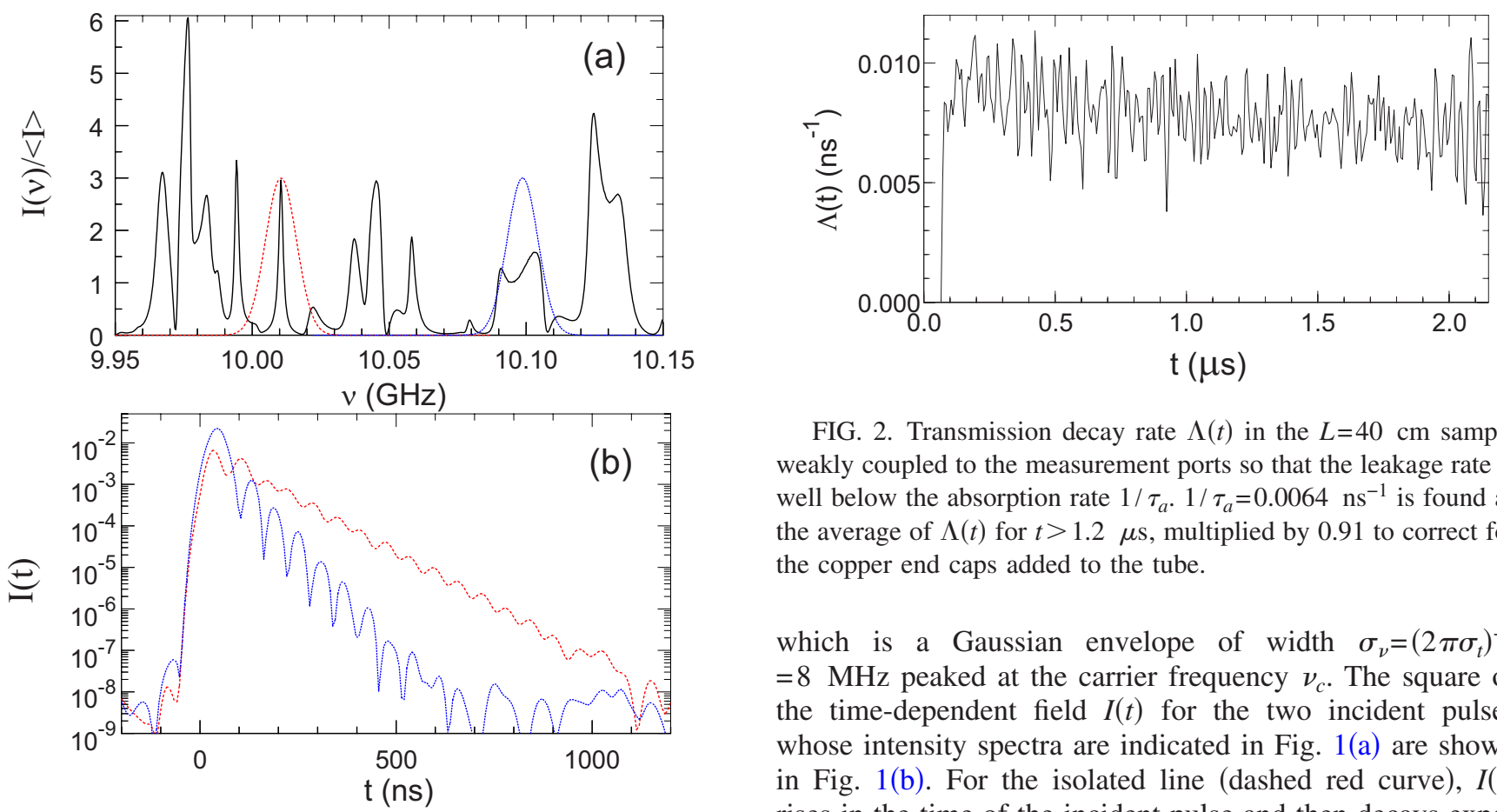

FIG. 1. (Color online) (a) Transmitted intensity spectrum (solid black curve) in a random sample of $L=40 \mathrm{~cm}$ and Gaussian spectra of incident pulses peaked at the center of the isolated line (dashed red curve) and overlapping lines (dotted blue curve). (b) Intensity responses to the Gaussian incident pulses with spectral functions shown in (a).

results indicate the greater prominence of overlapping modes in 1D samples than in quasi-1D samples at times of a few times $t_{p}$.

\section{EXPERIMENTS}

Microwave spectra of the field transmitted through lowdensity random mixtures of alumina spheres were taken with the use of a vector network analyzer. The wave is launched and detected by conical horns placed $30 \mathrm{~cm}$ in front of and behind the sample. Alumina spheres with diameter of 0.95 $\mathrm{cm}$ and index of refraction of 3.14 are embedded within Styrofoam shells to produce a sample with alumina volume fraction of 0.068 , which displays distinct sphere resonances. ${ }^{37}$ The sample is contained within a copper tube with diameter of $7.3 \mathrm{~cm}$ and thin plastic end pieces. Spectra are taken for 10000 configurations produced by briefly rotating the tube, in samples of lengths 13, 29, 40, 50, 61, and $90 \mathrm{~cm}$. Measurements are made just above the first sphere resonance over the frequency range of 9.95-10.15 GHz, in which the change in static and dynamic propagation parameters is small.

A typical transmission spectrum in a sample with $L$ $=40 \mathrm{~cm}$, obtained by squaring the corresponding field spectrum, $I(\nu)=|E(\nu)|^{2}$, is shown in Fig. 1(a). The response to a Gaussian incident pulse, $I_{0}(t) \sim \exp \left(-t^{2} / \sigma_{t}^{2}\right)$, with $\sigma_{t}=20 \mathrm{~ns}$, is obtained by taking the Fourier transform of the product of the field spectrum and the spectrum of the incident pulse,

FIG. 2. Transmission decay rate $\Lambda(t)$ in the $L=40 \mathrm{~cm}$ sample weakly coupled to the measurement ports so that the leakage rate is well below the absorption rate $1 / \tau_{a} .1 / \tau_{a}=0.0064 \mathrm{~ns}^{-1}$ is found as the average of $\Lambda(t)$ for $t>1.2 \mu \mathrm{s}$, multiplied by 0.91 to correct for the copper end caps added to the tube.

which is a Gaussian envelope of width $\sigma_{\nu}=\left(2 \pi \sigma_{t}\right)^{-1}$ $=8 \mathrm{MHz}$ peaked at the carrier frequency $\nu_{c}$. The square of the time-dependent field $I(t)$ for the two incident pulses whose intensity spectra are indicated in Fig. 1(a) are shown in Fig. 1(b). For the isolated line (dashed red curve), $I(t)$ rises in the time of the incident pulse and then decays exponentially with decay rate $\Gamma=0.012 \mathrm{~ns}^{-1}$, which is essentially governed by the full width at half maximum (FWHM) Lorentzian linewidth, $\delta \nu_{E}=\Gamma / 2 \pi=1.9 \mathrm{MHz}$. For the overlapping lines (dotted blue curve), $I(t)$ shows structure due to the beating of the underlying modes. $\langle I(t)\rangle$ is obtained by subtracting from the average of the measured time response the constant background at long times which results from noise in the measurement of $I(\nu)$. To compensate for losses due to absorption, and thus to facilitate the comparison of the measurements to dynamical models of localization, $\langle I(t)\rangle$ is multiplied by $\exp \left(t / \tau_{a}\right)$, where $1 / \tau_{a}$ is the absorption rate. $1 / \tau_{a}=0.0064 \mathrm{~ns}^{-1}$ is found from the decay rate of transmission in a $40-\mathrm{cm}$-long sample with copper end caps, which is weakly coupled to the measurement ports, so that the leakage rate is well below the absorption rate (Fig. 2).

\section{THEORETICAL APPROACHES}

We next describe the two theoretical approaches used to describe pulse transmission, which are (1) SCLT and (2) DSPS model.

(1) In an open system, the SCLT developed by VW (Ref. 28) can be generalized as follows:

$$
\frac{1}{D(z, \Omega)}=\frac{1}{D_{B}}\left[1+\frac{v_{E}}{2 N} G(z, z ; \Omega)\right],
$$

where $D_{B}=v_{E} \ell / 3$ is the Boltzmann diffusion constant, $D(z, \Omega)$ is the renormalized diffusion coefficient, $N$ is the number of transverse modes, and $v_{E}$ is the transport velocity. The diagonal intensity Green's function $G(z, z ; \Omega)$ represents the return probability at $z$ and can be obtained from the following generalized diffusion equation: ${ }^{30}$ 


$$
\partial_{z}\left[D(z, \Omega) \partial_{z} G\left(z, z^{\prime} ; \Omega\right)\right]+i \Omega G\left(z, z^{\prime} ; \Omega\right)=-\delta\left(z-z^{\prime}\right),
$$

with mixed boundary conditions at both ends, $z_{0} D\left(z_{b} ; \Omega\right) \partial_{z} G\left(z_{b}, z^{\prime} ; \Omega\right) \mp D_{B} G\left(z_{b}, z^{\prime} ; \Omega\right)=0$, where $z_{b}=0$ or $L, z_{0}=(2 / 3) \ell(1+R) /(1-R)$ is the extrapolation length, and $R$ is the internal reflection coefficient. ${ }^{21,38,39}$ Equations (1) and (2) are solved self-consistently in real $\Omega$ space to obtain $G\left(z, z^{\prime} ; \Omega\right)$. The intensity just outside the output surface, $\langle I(t)\rangle$, is obtained by taking the Fourier transform of $G\left(L, z^{\prime}=\ell ; \Omega\right)$ in $\Omega$. In our calculations, we use the values of $N=32$, which is the number of propagating modes in the waveguide at $10 \mathrm{GHz}$, and of $v_{E}=11.85 \mathrm{~cm} / \mathrm{ns}$ calculated $^{40}$ for a sample with a volume fraction of alumina spheres of 0.068 just above the first Mie resonance at $10 \mathrm{GHz}$. The values of $\ell$ and $R$ will be determined by fitting the calculated $\langle I(t)\rangle$ to the measured data. Equations (1) and (2) are derived in Appendix B.

(2) Because transmission at long times may be determined by the longitudinal structure of spectrally isolated localized modes, we propose here a DSPS model to treat the statistics of such modes. In one dimension, the steady-state intensity at $z=L$ of resonantly excited modes relative to the incident wave at $z=0_{+}$, for modes peaked a distance $z$ from either sample boundary, was given by Azbel, ${ }^{3} T=\exp [-2 \gamma$ $(L-2 z)]$ for $0<z<L / 2$. The Lyapunov exponent $\gamma=1 / 2 \xi$ is drawn from a Gaussian distribution,

$$
P(\gamma)=\sqrt{L / 2 \pi \bar{\gamma}} \exp \left[-(\gamma-\bar{\gamma})^{2} /(2 \bar{\gamma} / L)\right],
$$

according to the SPS hypothesis. ${ }^{36}$ Here we generalize the SPS theory to study the dynamics of transmitted waves through isolated localized modes. We assume that the position of peak intensity for the modes is uniformly distributed between 0 and $L$. The decay rate of localized states is the ratio of the sum of the outgoing fluxes at the open ends to the integrated wave energy inside the sample,

$$
\Gamma(\gamma, z)=\beta v_{E} \frac{1+\exp [-2 \gamma(L-2 z)]}{\{2 \exp (2 z \gamma)-\exp [-2 \gamma(L-2 z)]-1\} / 2 \gamma} .
$$

The coupling factor $\beta$ reflects the reduction in the transmitted flux due to the angular spread about the normal to the interface, as well as the angular average of internal reflection at the interface, $R$, and by the character of transport near the boundary. The transmitted intensity is then

$$
\langle I(t)\rangle=\frac{1}{2 L} \int_{2 / L}^{\infty} d \gamma \int_{0}^{L / 2} d z P(\gamma) T \Gamma^{2} \exp (-\Gamma t) .
$$

The factor $T(\Gamma / 2)^{2} \exp (-\Gamma t)$ is the square of the Fourier transform of a Lorentzian line in the field spectrum with linewidth $\Gamma / 2 \pi$. Internal reflection does not change $T$ appreciably since the intensity throughout the sample is enhanced by the same factor that inhibits transmission at the interface.

\section{RESULTS AND DISCUSSIONS}

The early-time behavior of the measured $\langle I(t)\rangle$ in the 61$\mathrm{cm}$-long sample is shown as the black curve in Fig. 3(a). In
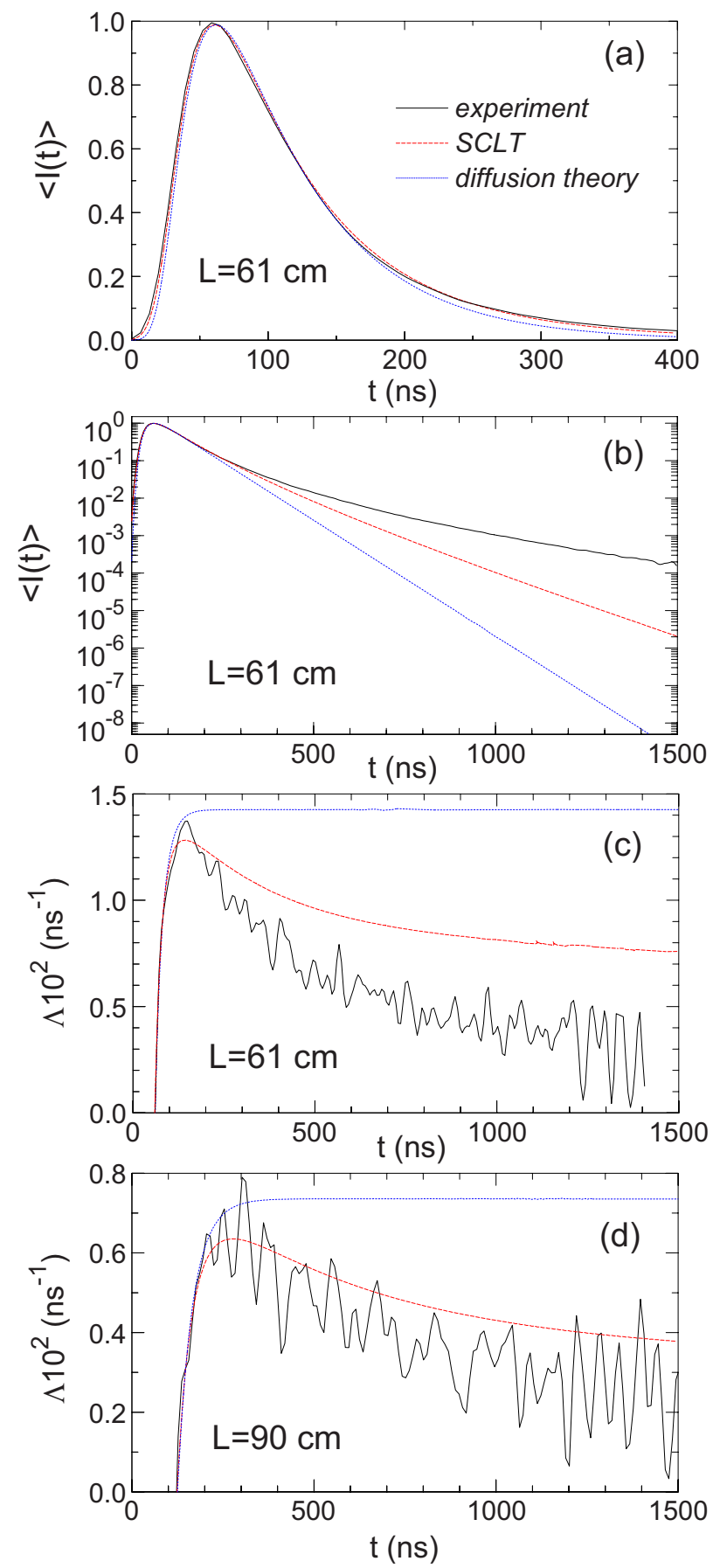

FIG. 3. (Color online) (a) Fit of SCLT (dashed red curve) at early times to the average intensity response (solid black curve) to a Gaussian pulse with $\sigma_{\nu}=15 \mathrm{MHz}$ in the $L=61 \mathrm{~cm}$ sample and the result of classical diffusion theory (dotted blue curve); (b) semilogarithmic plot of $\langle I(t)\rangle$ including longer times; the measured and calculated decay rates $\Lambda(t)$ for the samples with (c) $L=61 \mathrm{~cm}$ and (d) $L=90 \mathrm{~cm}$. In (a) and (b), the curves are normalized to the peak value.

order to compare this result with SCLT, we fit $\langle I(t)\rangle$ of SCLT to the measured data shown in Fig. 3(a) to determine the values of the transport mean free path $\ell$ and the internal reflection coefficient $R$, which are the only fitting parameters. The fit gives $\ell \approx 2.0 \mathrm{~cm}$ and $R \approx 0.64$, and therefore $D_{B}$ 


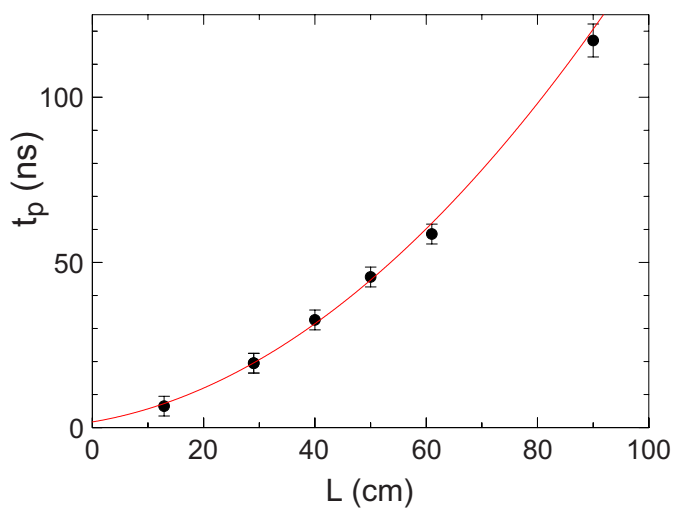

FIG. 4. (Color online) Comparison of the measured delay of the intensity peak in alumina samples (circles) from the peak of an incident Gaussian pulse of $\sigma_{\nu} \ll \tau_{D}^{-1}$ with the prediction of diffusion theory, $t_{p}=0.89 \tau_{D}$ (solid red curve).

$=7.9 \mathrm{~cm}^{2} / \mathrm{ns}, z_{0}=6.1 \mathrm{~cm}$, and $\tau_{D}=70.4 \mathrm{~ns}$. The fit is shown as the dashed red curve in Fig. 3(a). Excellent agreement is found between SCLT and experiment for $t<4 \tau_{D}$. To compare the long-time behavior, we present these results in a semilogarithmic plot in Fig. 3(b). In Figs. 3(a) and 3(b), we also plot the result of classical diffusion theory ${ }^{41}$ (dotted blue curve), by using the values of $R$ and $D_{B}$ obtained from the fit above, in which localization effects are absent. The surprising agreement between classical diffusion, SCLT, and measurement for $t<2 \tau_{D}$ suggests that the renormalization of the diffusion coefficient does not change significantly for early times following the rapid rise of the transmitted pulse, even for the localized waves studied here. Renormalization of the diffusion constant becomes noticeable for $t>2 \tau_{D}$.

The applicability of classical diffusion theory at early times can also be seen in the excellent agreement between the measured and calculated peak arrival times $t_{p}$ for six sample lengths shown in Fig. 4. We believe that diffusion theory is applicable to the early-time pulse transmission in localized samples because the following inequalities are satisfied in our measurements: $\tau_{D}<\tau_{H}<\tau_{\mathrm{Th}}$, where $\tau_{D}$ is a measure of the peak arrival time of a pulse diffusing through a sample, $t_{p} \sim \tau_{D}$. The first inequality, $\tau_{D}<\tau_{H}$, guarantees that wave propagation is diffusive for early times. Since $\tau_{H}$ is the time that would be required for a wave to visit each coherence volume of the sample, the probability of a wave revisiting a coherence volume through which it has passed is less than unity for $\tau_{D}<t<\tau_{H}$, and wave interference does not strongly modify average transport. As a result, propagation is nearly diffusive. But since $\tau_{H}<\tau_{\mathrm{Th}}, g<1$, and $\operatorname{var}\left(s_{a}\right)$ $>2 / 3$, waves are localized in the sample. Thus, our localized samples are in the region of $1 / \pi^{2}<g<1$. For example, for the $L=61 \mathrm{~cm}$ sample we have $\tau_{D}=70.4 \mathrm{~ns}, \tau_{H}=528 \mathrm{~ns}$, and $\tau_{\mathrm{Th}}=695 \mathrm{~ns}$, whereas for the $L=90 \mathrm{~cm}$ sample $\tau_{D}$ $=136.3 \mathrm{~ns}, \tau_{H}=780 \mathrm{~ns}$, and $\tau_{\mathrm{Th}}=1346 \mathrm{~ns}$. The values of $\tau_{H}$ are obtained from measurements of the average spacing between modes in a closed sample. ${ }^{37}$

It should be pointed out that in the quasi-1D system studied here, the radius of the tube is comparable to $\ell$. This is different from the three-dimensional (3D) system studied in Ref. 34, in which the wave was not confined in the transverse plane. We also note that the values of $t_{p}$ shown in Fig. 4 do not extrapolate to zero as $L$ approaches zero. This is because the intensity at the sample interface with air does not vanish. Rather the intensity in diffusive samples follows a diffusion equation in the bulk of the sample with a boundary condition that the intensity extrapolates to zero a distance $z_{0}$ beyond the sample. In our samples, we find $t_{p}=0.89\left(L+2 z_{0}\right)^{2} / \pi^{2} D_{B}$ and $t_{p}$ extrapolates to zero at $L=-2 z_{0}$.

Since the SCLT includes localization effects, it gives a decay in $\langle I(t)\rangle$ which is slower than exponential, as seen in Fig. 3(b). However, for $t>4 \tau_{D}$, SCLT does not fully account for the localization effects and gives a faster decay rate than is measured. To show this more clearly, we plot both the measured and calculated decay rates $\Lambda(t)$ in Figs. 3(c) and 3 (d) for the samples with $L=61 \mathrm{~cm}$ and $L=90 \mathrm{~cm}$. The peak positions shown in Figs. 3(a) and 3(b) correspond to $\Lambda(t)$ $=0$ in Figs. 3(c) and 3(d), respectively. Also plotted in Figs. 3(c) and 3(d) are the results of the classical diffusion theory (dotted blue lines). It is also seen that for a given delay time, a larger deviation is found for the $L=61 \mathrm{~cm}$ sample than for the $L=90 \mathrm{~cm}$ sample which is deeper in the localization regime. This is because the average number of times a path returns to a coherence volume is larger in the shorter sample for a given time delay, since the path lengths within the samples are identical, while the sample volume is smaller for the shorter sample. Thus the number of closed loops and the consequent renormalization are greater in the shorter sample. A slower decay in both samples suggests that more extended quasimodes, which may exhibit diffusionlike behavior and may be adequately described by SCLT, have largely decayed, so that the wave is transmitted increasingly via long-lived localized modes. Such isolated modes, as well as overlapping modes, are observed in the intensity spectra shown in Fig. 1(a). Thus, it would be interesting to compare the measured $\langle I(t)\rangle$ at long times with the DSPS model. Since the DSPS model is a $1 \mathrm{D}$ model, we can check the validity of this model by comparing to results of $1 \mathrm{D}$ simulations.

The 1D systems considered here are random samples of $L / a$ layers of equal thickness $a$, embedded in air with $\epsilon=1$ and wave speed $c$, the speed of light. The dielectric constant in each layer is a random number between 0.3 and 1.7 , drawn from a uniform distribution. Thus, the random medium has averaged dielectric constant $\bar{\epsilon}=1$, which is equal to the dielectric constant of the surrounding medium. This assures that internal reflections at the sample boundaries do not introduce effects above and beyond scattering within the sample. $^{42}$ The average localization length is calculated through $\bar{\xi}=-L /\langle\ln T\rangle$, where the static transmission coefficient $T$ is calculated by using transfer-matrix method ${ }^{43}$ and averaging over 1000 configurations. The calculated spectrum of $\bar{\xi}$ in terms of a dimensionless angular frequency is shown in Fig. 5. In our dynamic study, we use a Gaussian incident pulse with carrier frequency $\omega_{0}=1.65 \mathrm{c} / \mathrm{a}$ and width $\sigma$ $=0.14 c / a$, and the time-varying intensity just beyond the output surface is calculated. Over the width of the incident spectrum, $1.50 c / a<\omega<1.80 c / a$, the localization length $\bar{\xi}$ $=22 a$ is relatively unchanged, as seen in the inset of Fig. 5 . $\langle I(t)\rangle$ is obtained by averaging over 10000 configurations. Since $R=0$ and no angle average is necessary, we take $\beta$ 


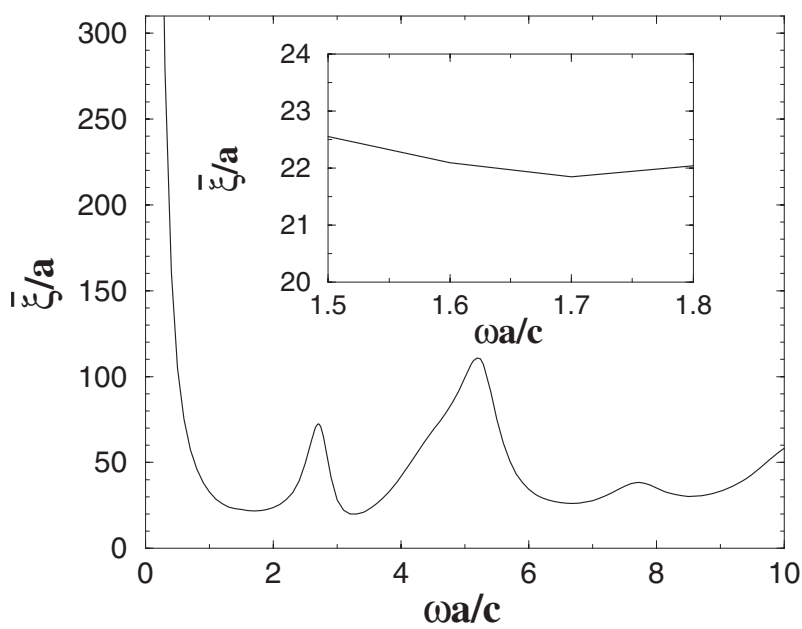

FIG. 5. Localization length of a 1D system of layers of thickness $a$ with random values of $\epsilon$ plotted versus normalized angular frequency, as determined from the calculated static transmission coefficient.

$=1$. Further, we assume $v_{E}=c$ for the effective medium velocity, since there are no resonances with individual elements of the sample over the pulse bandwidth.

In choosing the values of $L$ in 1D simulations, we take advantage of the scaling property of the DSPS model, which allows us to compare the decay rates $\Lambda(t)$ of quasi-1D systems to $1 \mathrm{D}$ systems with the same values of $L / \bar{\xi}$. This is done by introducing the dimensionless time $\tau \equiv \bar{\xi} / \beta v_{E}$ and recasting $\Gamma t$ of Eq. (4) into $(\Gamma \tau)(t / \tau)$, where the dimensionless decay rate $\Gamma \tau \equiv \widetilde{\Gamma}$ can be written $[$ see Eq. (3)] in the form

$$
\widetilde{\Gamma}(\tilde{\gamma}, \tilde{z} ; L / \bar{\xi})=\tilde{\gamma} \frac{1+\exp [-2 \widetilde{\gamma}(L / \bar{\xi})(1-2 \widetilde{z})]}{(2 \exp \{2(\widetilde{z} \widetilde{\gamma})-\exp [-2 \widetilde{\gamma}(L / \bar{\xi})(1-2 \widetilde{z})]-1\})},
$$

where $\tilde{\gamma} \equiv \gamma / \bar{\gamma}$ and $\tilde{z} \equiv z / L$. It is clearly seen from Eq. (5) that the dimensionless decay rate $\widetilde{\Gamma}$, and hence $\widetilde{\Lambda}(t / \tau) \equiv \Lambda \tau$, becomes scaling functions of $\gamma / \bar{\gamma}$ and $z / L$ for any fixed value of $L / \bar{\xi}$, independent of whether the system is $1 \mathrm{D}$ or quasi-1D. Thus, Eq. (5) allows us to compare the decay rates $\tilde{\Lambda}$ of quasi-1D systems with 1D systems, as long as they have the same value of $L / \bar{\xi}$. The comparison does not depend on the detailed characteristics of the sample such as the layer thickness or the nature of randomness.

1D simulations were carried out for two sample lengths which satisfy the scaling condition that $L / 22 a=61 / 30$ and $90 / 30$. Here we have used the measured value of $\bar{\xi}=30 \mathrm{~cm}$ obtained from $\operatorname{var}\left(s_{a b}\right)$, as shown in Appendix A. The results of $\widetilde{\Lambda}(\Lambda \tau)$ are plotted as functions of $t / \tau$ in Figs. 6(a) and 6(b). Figure 6 shows that our 1D simulation results are in good agreement with the predictions of DSPS model at long times. This demonstrates the validity of DSPS and of the scaling arguments we have used. For a different 1D system, the localization length would change to a value different
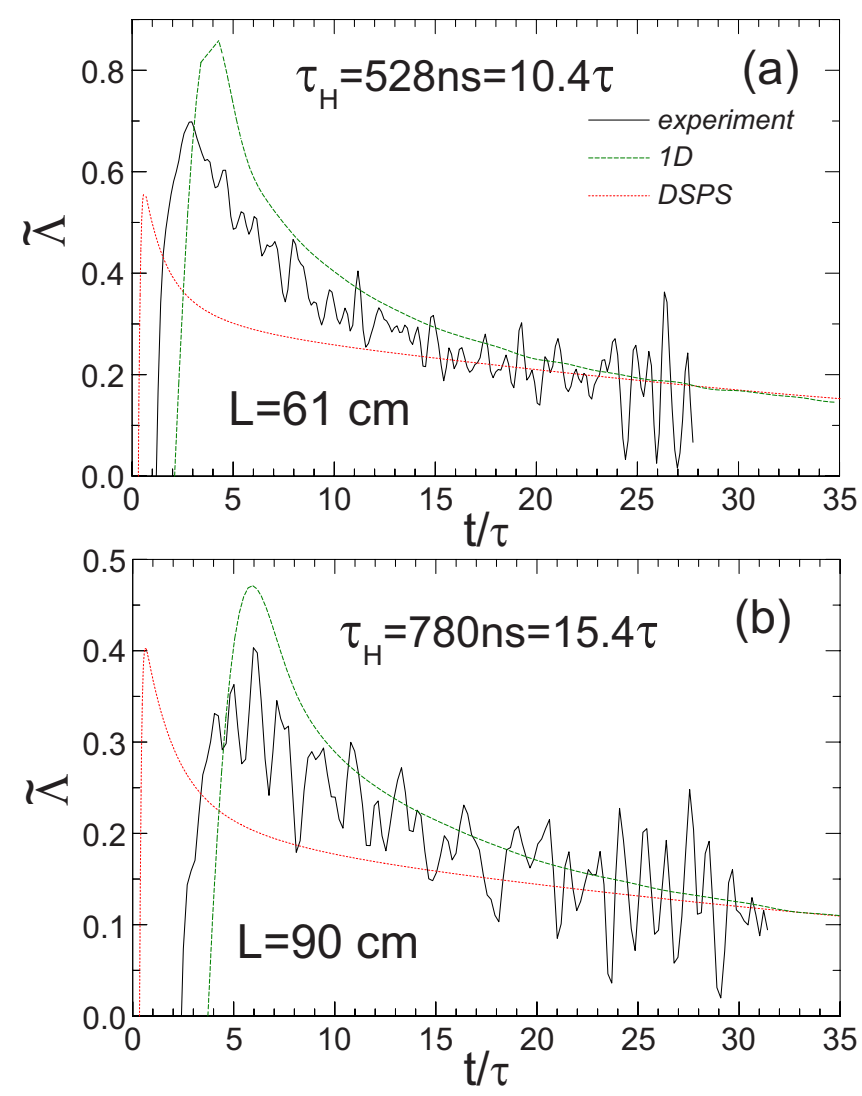

FIG. 6. (Color online) Decay rates in samples with (a) $L$ $=61 \mathrm{~cm}$ and (b) $90 \mathrm{~cm}$ are compared to predictions of DSPS model (dotted red curve). $\tau_{D}=70.4 \mathrm{~ns}$ and $\tau_{H}=528 \mathrm{~ns}$ for $L=61 \mathrm{~cm}$, and $\tau_{D}=136.3 \mathrm{~ns}$ and $\tau_{H}=780 \mathrm{~ns}$ for $L=90 \mathrm{~cm}$. The comparison to 1D simulations (dashed green curve) is made by using the dimensionless decay rate $\widetilde{\Lambda}=\tau \Lambda$, plotted as a function of the dimensionless time $t / \tau$, where $\tau=\bar{\xi} / \beta v_{E}$.

from $\bar{\xi}=22 a$, but the value of $\tau$ used in the dimensionless time would also change, so that wave dynamics in the scaled DSPS theory in units of $\tau$ would remain the same.

To compare these results with the decay rates of the quasi-1D systems, we fit $\Lambda(t)$ obtained from Eq. (4) with $\bar{\xi}$ $=30 \mathrm{~cm}$ to the measured decay rates in Figs. 3(c) and 3(d) at long times, to find $\beta \approx 0.05$. This value depends only weakly upon the value of $\bar{\xi}$; it does not change even when $\bar{\xi}$ is varied by $10 \%$. The dimensionless decay rates $\tilde{\Lambda}$ are plotted as functions of $t / \tau$ in Figs. 6(a) and 6(b). The quasi-1D and 1D results merge at long times for both the 61- and the $90-\mathrm{cm}-$ long samples.

The small coupling factor $(\beta=0.05)$ at the boundary found in the quasi-1D system is at least in part a consequence of strong reflection of waves propagating off the normal to the surface in quasi-1D samples. This is reminiscent of total internal reflection beyond the critical angle in samples with smooth interfaces. The rise time of transmission in the DSPS model is essentially the rise time of localized modes, which is the rise time of the incident pulse. Thus the slower rise observed in the experiment indicates the dominance of overlapping (as opposed to isolated) modes. At long times, $t>\tau_{H}$ (or $\left.t / \tau>\tau_{H} / \tau\right)$, however, the agreement of 
the DSPS model with measurements indicates that the energy within the sample is stored in long-lived localized modes. It should be mentioned that what we have considered here is the decay rate, not $\langle I(t)\rangle$, which can only be described if overlapping states are included in the model. Since the DSPS model does not include necklace states, it cannot give a complete description of $\langle I(t)\rangle$, particularly at early times. We note further that though states in finite samples are not strictly localized since there is some degree of hybridization of resonances within the sample, states may still be localized in an effective sense with only weak admixtures of neighboring states.

The longer delay of the peak in $\tau \Lambda$ and the faster decay rate after the peak found in $1 \mathrm{D}$ simulations relative to measurements in the quasi-1D samples, seen in Fig. 6, indicate that overlapping quasiextended modes ${ }^{23-25}$ which produce diffusionlike behavior at early times are more prominent in one dimension than in quasi-one-dimension. Since the localization length is approximately equal to the transport mean free path in random 1D samples, there does not exist a clear region of diffusion in one dimension. The larger decay rate found in the simulations at early times is due to the overlap of modes, which results in necklace states rather than in strict diffusion. At longer times, most of the energy stored in relatively short-lived necklace states has leaked out and what remains is the energy stored in spectrally isolated, long-lived localized modes. As a result, the decay rate predicted by the DSPS model accurately describes transmission in 1D and quasi-1D samples at long times.

\section{CONCLUSIONS}

In conclusion, we have observed the evolution in time of the nature of pulsed transmission of localized waves in quasi-1D samples. At short times, $t<2 \tau_{D}$, propagation is described by Boltzmann diffusion and the renormalization of diffusion constant is insignificant. At intermediate times, $2 \tau_{D}<t<4 \tau_{D}$, transport is described in terms of a positionand frequency-dependent renormalized diffusion coefficient, while at later times, $t>\tau_{H}$, energy flows predominantly from isolated localized modes and the decay rate can be described by the DSPS model, which coincides with results for $1 \mathrm{D}$ simulations once the corresponding samples are appropriately scaled. The evolving dynamics mirrors a change in the modal distribution of energy with time. At early time, the transmitted flux is primarily associated with short-lived overlapping modes, while at longer times, transmission derives from long-lived localized modes. As a consequence, transport for localized waves may be diffusive at early times, while transport even for diffusive waves may be suppressed at long times. This work shows that a unified theoretical description of pulsed propagation of localized waves will need to incorporate a full description of mode overlap, which would include the distribution of spacings and widths of quasimodes of the random medium as a function of the average overlap parameter, $\delta=\delta \nu / \Delta \nu$.

\section{ACKNOWLEDGMENTS}

We thank P. W. Brouwer for communication of the rela- tion between variance of total transmission and localization length. This research was sponsored by the National Science Foundation under Grant No. DMR0538350 and by the Hong Kong RGC under Grant No. 604506.

\section{APPENDIX A: VARIANCE OF THE TOTAL TRANSMISSION}

It can be shown that the variance of normalized transmission, $\operatorname{var}\left(s_{a}\right)$, can be expressed in terms of a single parameter, $\zeta=L / \bar{\xi}$, and therefore can be used to determine the localization length $\bar{\xi}$ of a random sample. ${ }^{44}$ In the context of random matrix theory of quantum transport, the average and the variance of the total transmission $T_{a}$ are given, respectively, by

$$
\left\langle T_{a}\right\rangle=N^{-1}\langle T\rangle \text { and } \operatorname{var}\left(T_{a}\right)=N^{-2}\left[\operatorname{var}(T)+\left\langle\operatorname{tr}\left(t^{\dagger} t\right)^{2}\right\rangle\right]
$$

where $T=\operatorname{tr}\left(t^{\dagger} t\right)$ is the conductance (transmittance). Now we make use of an identity that can be derived from DMPK equation (see, for example, Refs. 45 and 46),

$$
\frac{\partial}{\partial \zeta}\langle T\rangle=-\left\langle T^{2}\right\rangle-\left\langle\operatorname{tr}\left(t^{\dagger} t\right)^{2}\right\rangle .
$$

Combining Eqs. (A1) and (A2), we find

$$
\operatorname{var}\left(s_{a}\right)=-\langle T\rangle^{-2} \frac{\partial}{\partial \zeta}\langle T\rangle-1 .
$$

The average $T$ in the orthogonal ensemble was computed by Mirlin et al. ${ }^{47}$ as follows:

$$
\begin{aligned}
& \langle T\rangle=\frac{\pi}{4} \int_{0}^{\infty} d \lambda \tanh ^{2}(\pi \lambda / 2)\left(1+\lambda^{2}\right)^{-1} P(1, \lambda, \lambda) T(1, \lambda, \lambda, \zeta) \\
& +8 \sum_{l=3,5, \ldots}^{\infty} \int_{0}^{\infty} d \lambda_{1} \int_{0}^{\infty} d \lambda_{2} l\left(l^{2}-1\right) \lambda_{1} \tanh \left(\pi \lambda_{1} / 2\right) \lambda_{2} \\
& \times \tanh \left(\pi \lambda_{2} / 2\right) P\left(l, \lambda_{1}, \lambda_{2}\right) T\left(l, \lambda_{1}, \lambda_{2}, \zeta\right) \\
& \times \prod\left(-1+\sigma l+i \sigma_{1} \lambda_{1}+i \sigma_{2} \lambda_{2}\right)^{-1}, \\
& \sigma, \sigma_{1}, \sigma_{2}= \pm 1
\end{aligned}
$$

where

$$
\begin{gathered}
P\left(l, \lambda_{1}, \lambda_{2}\right)=l^{2}+\lambda_{1}^{2}+\lambda_{2}^{2}+1, \\
T\left(l, \lambda_{1}, \lambda_{2}, \zeta\right)=e^{-P\left(l, \lambda_{1}, \lambda_{2}\right) \zeta / 4} .
\end{gathered}
$$

The effects of localization and absorption are intertwined in steady-state measurements. In order to obtain the values of $\operatorname{var}\left(s_{a}\right)$ that would be measured in the absence of absorption, the field spectra are Fourier transformed to give the response to a narrow Gaussian pulse in the time domain. To compensate for losses due to absorption, the time-dependent field is multiplied by $\exp \left(t / 2 \tau_{a}\right)$, where $t$ is the time delay from the incident pulse and $1 / \tau_{a}=0.0064 \mathrm{~ns}^{-1}$ is the absorption rate (see Fig. 2). This new field is transformed back to the frequency domain. Intensity spectra and the variance of intensity are then computed. The values of $\operatorname{var}\left(s_{a}\right)$ found from $\operatorname{var}\left(s_{a}\right)=\left[\operatorname{var}\left(s_{a b}\right)-1\right] / 2$ and $\bar{\xi}$ determined from Eq. (A3) for 
TABLE I. Values of $\operatorname{var}\left(s_{a}\right), L / \bar{\xi}$, and $\bar{\xi}$ in alumina samples, as found from Eq. (A3).

\begin{tabular}{cccc}
\hline \hline$L(\mathrm{~cm})$ & $\operatorname{var}\left(s_{a}\right)$ & $L / \bar{\xi}$ & $\bar{\xi}(\mathrm{cm})$ \\
\hline 29 & 1.01 & 0.985 & 29.4 \\
40 & 1.84 & 1.40 & 28.6 \\
50 & 2.28 & 1.63 & 30.7 \\
61 & 2.83 & 1.83 & 33.4 \\
90 & 6.36 & 2.70 & 33.3 \\
\hline \hline
\end{tabular}

samples of lengths $29,40,50,61$, and $90 \mathrm{~cm}$ are listed in Table I. Notice that the accuracy of $\operatorname{var}\left(s_{a}\right)$ depends on the dynamic range of the time-dependent field, which decreases with increasing $L$ since more of the transmitted signal is lost due to absorption in longer samples. We expect therefore that the values of $\operatorname{var}\left(s_{a}\right)$ are smaller than would be obtained in a sample without absorption and that the error increases with $L$. This could explain a systematic increase in $\bar{\xi}$ with increasing $L$, seen in Table I. We conclude that the best estimate is $\bar{\xi}=30 \mathrm{~cm}$.

\section{APPENDIX B: DERIVATIONS OF EQS. (1) and (2)}

To derive Eqs. (1) and (2), we first use the known result of self-consistent localization theory for a 3D infinite medium derived by Kirkpatrick. ${ }^{48}$ Equation (4.8b) of Ref. 48 leads to the following self-consistent equation for the renormalized diffusion constant:

$$
\frac{1}{D(\Omega)}=\frac{1}{D_{B}}+\frac{3 \gamma}{4 \pi^{2} k^{3}} \int d \vec{q} \frac{1}{-i \Omega+D(\Omega) q^{2}},
$$

where $\gamma$ is the imaginary part of the self-energy. By using the relations $D_{B}=v_{E} \ell / 3$ and $\gamma(E)=k / \ell$, we can rewrite Eq. (B1) as

$$
\frac{1}{D(\Omega)}=\frac{1}{D_{B}}\left[1+\frac{v_{E}}{4 \pi^{2} k^{2}} \int d \vec{q} \frac{1}{-i \Omega+D(\Omega) q^{2}}\right],
$$

or

$$
\frac{1}{D(\Omega)}=\frac{1}{D_{B}}\left[1+\frac{2 \pi v_{E}}{k^{2}} G_{3 \mathrm{D}}(\vec{r}, \vec{r} ; \Omega)\right],
$$

where $G_{3 \mathrm{D}}(\vec{r}, \vec{r} ; \Omega)$ is the diagonal element of the following Green's function for the diffusion equation:

$$
G_{3 \mathrm{D}}\left(\vec{r}, \vec{r}^{\prime} ; \Omega\right)=\left(\frac{1}{2 \pi}\right)^{3} \int d \vec{q} \frac{e^{i \vec{q} \cdot\left(\vec{r}-\vec{r}^{\prime}\right)}}{-i \Omega+D(\Omega) q^{2}} .
$$

Moreover $G_{3 \mathrm{D}}\left(\vec{r}, \vec{r}^{\prime} ; \Omega\right)$ satisfies

$$
D(\Omega) \nabla^{2} G_{3 \mathrm{D}}\left(\vec{r}, \vec{r}^{\prime} ; \Omega\right)+i \Omega G_{3 \mathrm{D}}\left(\vec{r}, \vec{r}^{\prime} ; \Omega\right)=-\delta\left(\vec{r}-\vec{r}^{\prime}\right) .
$$

For quasi-1D samples studied here, we ignore weak localization effects in the transverse direction because the radius of the tube is comparable to $\ell$. In this case, the Green's function does not vary in the transverse direction and depends only upon $z$. For a tube of finite length, the renormalized diffusion constant also becomes a function of $z$, i.e., $D(z, \Omega) .{ }^{29}$ From the above considerations and Eq. (B5), the Green's function of a quasi-1D system satisfies the following generalized diffusion equation:

$$
\begin{aligned}
\partial_{z} & {\left[D(z, \Omega) \partial_{z} G_{\mathrm{Q} 1 \mathrm{D}}\left(z, z^{\prime} ; \Omega\right)\right]+i \Omega G_{\mathrm{Q} 1 \mathrm{D}}\left(z, z^{\prime} ; \Omega\right) } \\
& =-\frac{1}{A} \delta\left(z-z^{\prime}\right),
\end{aligned}
$$

where $A=\pi R^{2}$ is the cross-sectional area of the waveguide. Comparing Eq. (B6) with Eq. (2), we find $G_{\mathrm{Q} 1 \mathrm{D}}\left(z, z^{\prime} ; \Omega\right)$ $=(1 / A) G\left(z, z^{\prime} ; \Omega\right)$, where $G$ denotes the Green's function for $1 \mathrm{D}$ systems, i.e., Eq. (2). To find the self-consistent equation for quasi-1D systems, we replace $G_{3 \mathrm{D}}(\vec{r}, \vec{r} ; \Omega)$ in Eq. (B3) by $G_{\mathrm{Q} 1 \mathrm{D}}(z, z ; \Omega)=\left(1 / \pi R^{2}\right) G(z, z ; \Omega)$ and $D(\Omega)$ by $D(z, \Omega)$, yielding

$$
\frac{1}{D(z, \Omega)}=\frac{1}{D_{B}}\left[1+\frac{2 v_{E}}{(k R)^{2}} G(z, z ; \Omega)\right] .
$$

Equation (1) is finally obtained by using the relation $N$ $=(k R)^{2} / 4$ for the channel number of a cylindrical waveguide in Eq. (B7).

Below, we give an alternative derivation of Eq. (B3) by using the result of Sadakata, ${ }^{49}$ which is obtained from the self-consistent localization theory for tight-binding electrons. Equation (2.14) of Ref. 49 gives the following expression for the renormalization of conductivity:

$$
\frac{1}{\sigma(\Omega)}=\frac{1}{\sigma_{0}}\left[1+\frac{1}{\pi N_{F}} \sum_{\vec{q}} \frac{1}{D(\Omega) q^{2}-i \Omega}\right],
$$

where $N_{F}$ is the density of states (DOS) per site. By using the Einstein relation that relates the conductivity to the diffusion constant, Eq. (B8) can be rewritten as

$$
\frac{1}{D(\Omega)}=\frac{1}{D_{B}}\left[1+\frac{1}{\pi\left(N_{F} / a^{3}\right)} \frac{1}{a^{3}} \sum_{\vec{q}} \frac{1}{-i \Omega+D(\Omega) q^{2}}\right],
$$

where $a$ is the lattice constant and $N_{F} / a^{3}$ is the DOS per unit volume. For scalar waves in three dimensions, the DOS per unit volume has the form $\rho_{3 \mathrm{D}}=k^{2} /\left(2 \pi^{2} v\right) .{ }^{50}$ After replacing $N_{F} / a^{3}$ by $\rho_{3 \mathrm{D}}$ and the summation $\left(1 / a^{3}\right) \Sigma_{\vec{q}}$ by an integral $\left[1 /(2 \pi)^{3}\right] \int d \vec{q}$, Eq. (B9) becomes

$$
\frac{1}{D(\Omega)}=\frac{1}{D_{B}}\left[1+\frac{2 \pi v}{k^{2}} G_{3 \mathrm{D}}(\vec{r}, \vec{r} ; \Omega)\right] .
$$

Equation (B10) has the same form as Eq. (B3) if the phase velocity $v$ equals the energy transport velocity $v_{E}$, which is true in the long-wavelength limit. 
${ }^{1}$ P. W. Anderson, Phys. Rev. 109, 1492 (1958).

${ }^{2}$ Mesoscopic Phenomena in Solids, edited by B. L. Altshuler, P. A. Lee, and R. A. Webb (Elsevier Science, New York, 1991).

${ }^{3}$ M. Ya. Azbel, Phys. Rev. B 28, 4106 (1983); Solid State Commun. 45, 527 (1983).

${ }^{4}$ S. John, H. Sompolinsky, and M. J. Stephen, Phys. Rev. B 27, 5592 (1983); S. John, Phys. Rev. Lett. 53, 2169 (1984).

${ }^{5}$ A. Z. Genack, Phys. Rev. Lett. 58, 2043 (1987); A. Z. Genack and N. Garcia, ibid. 66, 2064 (1991).

${ }^{6}$ D. S. Wiersma, P. Bartolini, A. Lagendijk, and R. Righini, Nature (London) 390, 671 (1997).

${ }^{7}$ M. P. Van Albada and A. Lagendijk, Phys. Rev. Lett. 55, 2692 (1985).

${ }^{8}$ P. E. Wolf and G. Maret, Phys. Rev. Lett. 55, 2696 (1985).

${ }^{9}$ E. Akkermans, P. E. Wolf, and R. Maynard, Phys. Rev. Lett. 56, 1471 (1986).

${ }^{10}$ F. Scheffold, R. Lenke, R. Tweer, and G. Maret, Nature (London) 398, 206 (1999).

${ }^{11}$ S. Fiebig, C. M. Aegerter, W. Bührer, M. Störzer, E. Akkermans, G. Montambaux, and G. Maret, Europhys. Lett. 81, 64004 (2008).

${ }^{12}$ R. L. Weaver, Phys. Rev. B 47, 1077 (1993).

${ }^{13}$ A. A. Chabanov, M. Stoytchev, and A. Z. Genack, Nature (London) 404, 850 (2000).

${ }^{14}$ A. A. Chabanov, Z. Q. Zhang, and A. Z. Genack, Phys. Rev. Lett. 90, 203903 (2003).

${ }^{15}$ M. Störzer, P. Gross, C. M. Aegerter, and G. Maret, Phys. Rev. Lett. 96, 063904 (2006).

${ }^{16}$ D. J. Thouless, Phys. Rev. Lett. 39, 1167 (1977).

${ }^{17}$ A. Z. Genack, Europhys. Lett. 11, 733 (1990).

${ }^{18}$ M. Stoytchev and A. Z. Genack, Phys. Rev. Lett. 79, 309 (1997).

${ }^{19}$ E. Kogan and M. Kaveh, Phys. Rev. B 52, R3813 (1995).

${ }^{20}$ G. H. Watson, Jr., P. A. Fleury, and S. L. McCall, Phys. Rev. Lett. 58, 945 (1987); J. M. Drake and A. Z. Genack, ibid. 63, 259 (1989); A. Z. Genack and J. M. Drake, Europhys. Lett. 11, 331 (1990); P. M. Johnson, A. Imhof, B. P. J. Bret, J. G. Rivas, and A. Lagendijk, Phys. Rev. E 68, 016604 (2003).

${ }^{21}$ J. X. Zhu, D. J. Pine, and D. A. Weitz, Phys. Rev. A 44, 3948 (1991).

${ }^{22}$ A. D. Mirlin, Phys. Rep. 326, 259 (2000).

${ }^{23}$ J. B. Pendry, J. Phys. C 20, 733 (1987); Adv. Phys. 43, 461 (1994).

${ }^{24}$ P. Sebbah, B. Hu, J. M. Klosner, and A. Z. Genack, Phys. Rev. Lett. 96, 183902 (2006).

${ }^{25}$ J. Bertolotti, S. Gottardo, D. S. Wiersma, M. Ghulinyan, and L.
Pavesi, Phys. Rev. Lett. 94, 113903 (2005).

${ }^{26}$ S. K. Cheung, X. Zhang, Z. Q. Zhang, A. A. Chabanov, and A. Z. Genack, Phys. Rev. Lett. 92, 173902 (2004).

${ }^{27}$ B. L. Altshuler, V. E. Kravtsov, and I. V. Lerner, Sov. Phys. JETP 67, 795 (1988); B. A. Muzykantskii and D. E. Khmelnitskii, Phys. Rev. B 51, 5480 (1995).

${ }^{28}$ D. Vollhardt and P. Wölfle, Phys. Rev. B 22, 4666 (1980).

${ }^{29}$ B. A. van Tiggelen, A. Lagendijk, and D. S. Wiersma, Phys. Rev. Lett. 84, 4333 (2000)

${ }^{30}$ S. E. Skipetrov and B. A. van Tiggelen, Phys. Rev. Lett. 92, 113901 (2004).

${ }^{31}$ S. E. Skipetrov and B. A. van Tiggelen, Phys. Rev. Lett. 96, 043902 (2006)

${ }^{32}$ A. F. Ioffe and A. R. Regel, Prog. Semicond. 4, 237 (1960).

${ }^{33}$ C. M. Aegerter, M. Störzer, S. Fiebig, W. Bührer, and G. Maret, J. Opt. Soc. Am. A 24, A23 (2007).

${ }^{34}$ Z. Q. Zhang, A. A. Chabanov, S. K. Cheung, C. H. Wong, and A. Z. Genack, arXiv:0710.3155 (unpublished).

${ }^{35}$ Hefei Hu, A. Strybulevych, J. H. Page, S. E. Skipetrov, and B. A. van Tiggelen, Nat. Phys. 4, 945 (2008).

${ }^{36}$ P. W. Anderson, D. J. Thouless, E. Abrahams, and D. S. Fisher, Phys. Rev. B 22, 3519 (1980)

${ }^{37}$ A. A. Chabanov and A. Z. Genack, Phys. Rev. Lett. 87, 153901 (2001).

${ }^{38}$ A. Lagendijk, R. Vreeker, and P. de Vries, Phys. Lett. A 136, 81 (1989).

${ }^{39}$ J. H. Li, A. A. Lisyansky, T. D. Cheung, D. Livdan, and A. Z. Genack, Europhys. Lett. 22, 675 (1993).

${ }^{40}$ M. P. van Albada, B. A. van Tiggelen, A. Lagendijk, and A. Tip, Phys. Rev. Lett. 66, 3132 (1991).

${ }^{41}$ K. M. Yoo, F. Liu, and R. R. Alfano, Phys. Rev. Lett. 64, 2647 (1990).

${ }^{42}$ J. P. Fouque, J. Garnier, G. Papanicolaou, and K. Solna, Wave Propagation and Time Reversal in Randomly Layered Media (Springer, New York, 2007).

${ }^{43}$ Z. Q. Zhang, Phys. Rev. B 52, 7960 (1995).

${ }^{44} \mathrm{P}$. W. Brouwer (private communication).

${ }^{45}$ K. Frahm, Phys. Rev. Lett. 74, 4706 (1995).

${ }^{46}$ P. W. Brouwer and K. Frahm, Phys. Rev. B 53, 1490 (1996).

${ }^{47}$ A. D. Mirlin, A. Muller-Groeling, and M. R. Zernbauer, Ann. Phys. (N.Y.) 236, 325 (1994).

${ }^{48}$ T. R. Kirkpatrick, Phys. Rev. B 31, 5746 (1985).

${ }^{49}$ I. Sadakata, J. Phys. Soc. Jpn. 54, 736 (1985).

${ }^{50} \mathrm{P}$. Sheng, Introduction to Wave Scattering, Localization, and Mesoscopic Phenomena (Academic, New York, 1995). 